Literature Reviews

\title{
Ozone Therapy: Overview of its Potential Utility in Male Reproduction
}

\author{
${ }^{1,2}$ Zaher Merhi, ${ }^{3}$ Ali Bazzi, ${ }^{4}$ Rajean Moseley-LaRue, \\ ${ }^{4}$ Amber Ray Moseley, ${ }^{4}$ André Hugo Smith, ${ }^{2}$ John Zhang and ${ }^{5}$ Marco Ruggiero \\ ${ }^{1}$ Department of Biochemistry, Albert Einstein College of Medicine, Bronx, New York \\ ${ }^{2}$ New Hope Fertility Center, New York \\ ${ }^{3}$ St John Hospital and Medical Center, Detroit, MI, USA \\ ${ }^{4}$ HOCATT USA LLC, Weatherford, TX, USA \\ ${ }^{5}$ Silver Spring Sagl, Arzo-Mendrisio, Switzerland
}

Article history

Received: 06-12-2018

Revised: 21-12-2018

Accepted: 3-01-2019

Corresponding Author:

Zaher Merhi

Department of Biochemistry,

Albert Einstein College of

Medicine, Bronx, New York

Tel: (212) 517-7676

E-mail: zom00@hotmail.com

\begin{abstract}
Ozone (O3), a highly water-soluble inorganic molecule, is a gas made of three atoms of oxygen $(\mathrm{O})$ with a cyclic structure. Ozone can be produced by medical generators from pure oxygen after passing through a high voltage gradient. Ozone therapy (OT) can be given in medical practice via several routes that include transdermal, intramuscular, rectal, nasal, oral, vaginal, intravenous, intra-arterial, intraperitoneal, intra-pleural, topical, dental, intra-discal and by autohemotherapy. Because ozone, a highly reactive molecule, is a potent oxidant and anti-inflammatory agent, it has strong bactericidal, antiviral, anti-fungal and anti-protozoal actions as well as therapeutic effects on the immune system. With its multifaceted route of administration, OThas been used to treat several pathologies that involve the immune system such as cancers, sepsis, abscesses and chronic wounds, skin problems (such as eczema and psoriasis), HIV infection, asthma, arthritis, urologic problems, osteomyelitis and many others. The purpose of this review article is to evaluate the role of OT in the male reproductive system. We performed a review of all available basic science, experimental animal studies and clinical peer-reviewed articles published in PubMed and Google Scholar until November 2018. The literature so far retrieved shows that most studies pertaining to the effect of OT on male reproduction were performed in animals. Results to date show that OT, via improving the immune system, significantly protects testicular function in the setting of testicular torsion/ischemia, protects against the effect of gonadotoxic agents and treats bacterial infections in the semen. This article calls for a need for at least pilot studies in humans using OT in its safest route of administration, which is probably the transdermal one. This would be significant especially considering that male factor infertility constitutes up to one third of couple infertility and it is very common that poor semen parameters are irreversible with medical or surgical treatment, such as varicocele repair or vasectomy reversal.
\end{abstract}

Keywords: Ozone Therapy, Fertility, Male, Sperm, Testes, Transdermal

\section{Introduction}

Ozone (O3), a highly water-soluble inorganic molecule, is a gas made of three atoms of oxygen $(\mathrm{O})$ with a cyclic structure (Bocci, 2006; Smith et al., 2017) (Fig. 1). It is an unstable molecule due to its mesomeric state (Smith et al., 2017). The naturally occurring ozone is present in the stratosphere surrounding the earth (Cicerone, 1987) and protects humans from the dangerous ultraviolet radiations (Cicerone, 1987). Ozone can be produced by medical generators from pure oxygen after passing through a high voltage gradient. Because of its instability and short half-life $\left(40 \mathrm{~min}\right.$ at $\left.20^{\circ} \mathrm{C}\right)$, ozone cannot be stored in tanks (Smith et al., 2017). 


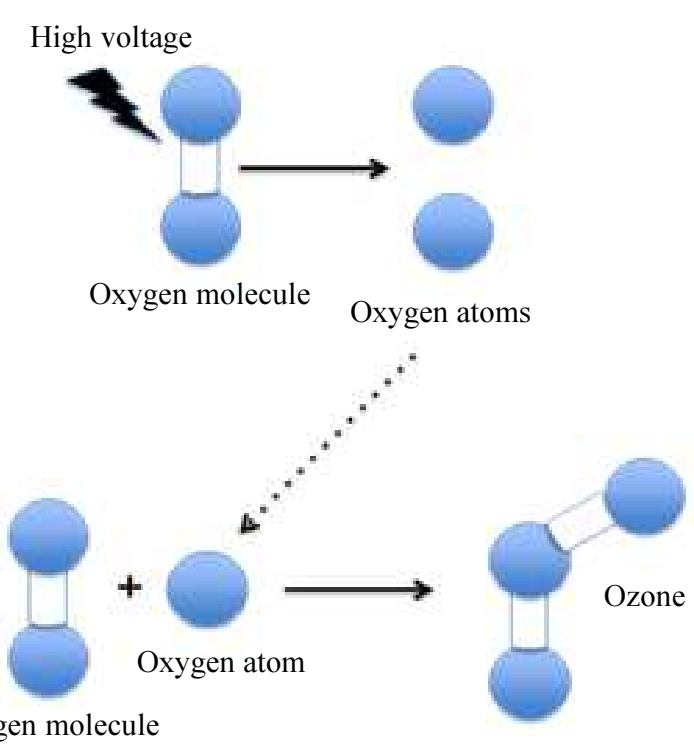

Oxygen molecule

Fig. 1: Molecular structure of ozone. Ozone is made up of three oxygen atoms, while the oxygen in the air is made up of only two atoms

Despites the difficulties with its storage, Ozone Therapy (OT) has been given in medical practice via several routes that include transdermal, intramuscular, rectal, nasal, oral, vaginal, intravenous, intra-arterial, intraperitoneal, intra-pleural, topical, dental, intra-discal and by auto-hemotherapy (Zanardi et al., 2016). In autohemotherapy, small amount of blood is collected from the patient, then exposed ex vivo to ozone then infused back into the patient (Smith et al., 2017). Because ozone is a potent oxidant and a highly reactive molecule, it has strong bactericidal, antiviral, anti-fungal and antiprotozoalactions (Duricic et al., 2015; Yamayoshi and Tatsumi, 1993; Sugita et al., 1992; Sato et al., 1990) as well as other therapeutic effects (Duricic et al., 2015; Yamayoshi and Tatsumi, 1993; Sugita et al., 1992; Sato et al., 1990; Clavo et al., 2018; Kashiwagi et al., 2001). Due to the advantage of its multifaceted route of administration, ozone has been used to treat several pathologies such as cancers (Clavo et al., 2018; Dogan et al., 2018), bad abscesses (Karatieieva et al., 2017; Li et al., 2018) and chronic wounds (Fitzpatrick et al., 2018), skin diseases[such as acne (Gloor and Lipphardt, 1976), eczema (Kosheleva and Kulikov, 2001) and psoriasis (Evstigneeva et al., 2018), HIV infection (Garber et al., 1991; Carpendale et al., 1993; Frankum and Katelaris, 1993; OPOI, 1994), fibromyalgia (Hidalgo-Tallon et al., 2013), asthma (Turner et al., 1989), inflammatory conditions (Kucukgul et al., 2016) including arthritis (Raeissadat et al., 2018; Manoto et al., 2018), cardiac conditions (Buyuklu et al., 2017), liver disorders (Safwat et al., 2014; Tezcan et al., 2018), eye disorders (Kaya et al.,
2017), urinary tract pathologies (Tasdemir et al., 2013), dyslipidemia (Di Paolo et al., 2005), osteomyelitis (Steinhart et al., 1999), Raynaud's disease (Cooke et al., 1997), sinusitis (Muminov and Khushvakova, 2002), dentistry diseases (Shamim, 2017), sepsis (Madej et al., 2007), musculoskeletal disorders (Seyam et al., 2018; Beyaz and Sayhan, 2018; Costa et al., 2018; Biazzo et al., 2018), diabetes complications (Kushmakov et al., 2018), neurologicdisorders (Wu et al., 2018; Yu et al., 2018; Resitoglu et al., 2018; $\mathrm{Hu}$ et al., 2018) and even nephropathy (Ozturk et al., 2018; Wang et al., 2018),

The mechanisms through whichOT works include the formation of hydrogen peroxide, areactive oxygen species (ROS), by reacting with polyunsaturated fatty acids and water (Bocci et al., 1998; Inal et al., 2011). Additionally, ozone forms a mixture of lipid ozonation products such as malondialdehyde (MDA) and ozonides (Inal et al., 2011). The mild oxidative stress caused by ozone leads to the activation of antioxidant cascades that in turn leads to production of Superoxide Dismutase (SOD), catalase and glutathione S-transferase (Bocci et al., 1998; Inal et al., 2011). Researchers and experts have argued for decades the value of OT in several medical conditions and it seems that there is a consensus on the concept that knowing the exact dose and duration of OT for each particular condition is critically needed in order to achieve optimal antioxidant activity (Bocci, 2006; Bocci et al., 1998; Bocci, 2008; 2007). The purpose of this review article is to evaluate the current literature pertaining to the role of OT in the male reproductive system.

\section{Methods Search Strategy and Data Extraction}

We performed a review of all available basic science, experimental animal studies and clinical peerreviewed articles published in the databases PubMed and Google Scholar until November 2018. Data were extracted from the text of the manuscripts, tables and graphs in those manuscripts. Keywords for searches were: "Ozone and male reproduction," "ozone and sperm," and "ozone and testes."

\section{Results}

\section{Effect of Ozone in Testicular Injury Caused by Testicular Torsion}

Testicular torsion is a urological problem caused by a twist of the spermatic cord. It constitutes a cause for male infertility due to testicular Ischemia/Reperfusion (I/R) and production of ROS due to Torsion and Detorsion (T/D) (Shimizu et al., 2016; Dokmeci, 2006). A study (Tusat et al., 2017) in rats $(\mathrm{n}=24)$ investigated the effect of OT on testicular damage due to $I / R$ by assessing biochemical parameters such as Ischemia Modified Albumin (IMA), Total Antioxidant Status (TAS), Total Oxidant Status (TOS) and Oxidative Stress 
Index (OSI, calculated by TOS: TAS ratio).Additionally, testicular tissue injury was scored using Johnsen Score by performing testicular biopsy. Rats were assigned to T/Dgroup, T/D + OT group, or control (sham procedure by making a scrotal incision). The torsion was performed on the left testis by rotating it 720 degrees clockwise to obtain torsion and maintained by fixing the testes. In the T/D group, detorsion was performed after $2 \mathrm{~h}$ of torsion and then maintained for $24 \mathrm{~h}$. The T/D + OT received $1 \mathrm{mg} / \mathrm{kg}$ intraperitonealozone immediately prior to detorsion for $2 \mathrm{~h}$. The results of that study showed that IMA, TOS and OSI were significantly higher in the T/D group compared to the control group. These levels were significantly reduced in the $\mathrm{T} / \mathrm{D}+\mathrm{OT}$ group. There were no significant differences in TAS levels among the 3 groups. As for the histopathologic score, the T/D group had seminiferous tubule germinal epithelia structure completely poured, spermatozoa were not available in the lumen and vasoconstriction was partly observed in inter-tubular area. However, compared to the T/D group, the T/D + OT group had more regular seminiferous tubule epithelial structure, had regular alignment in the lumen of the germinal epithelial cells and had partly observed spermatozoa. These findings suggest that OT was able to reduce the oxidative stress markers that usually rise in testicular torsion and significantly reduced scores that typically increase as a result of testicular torsion.

Another study (Mete et al., 2017), compared the efficacy of intra-testicular ozone with intraperitoneal ozone treatment in rats for the prevention of $I / R$ injury. Three-month old male Wistar rats $(\mathrm{n}=24)$ were divided into T/D group, T/D + intraperitoneal ozone group, or T/D + intra-testicular ozone group. Ozone was given at 4 $\mathrm{mg} / \mathrm{kg}$. Unilateral testicular torsion created by rotating left testis 720 degrees clockwise and fixing with hemiscrotum by a silk suture. The spermatic cord was detorsed after $1 \mathrm{~h}$ and reperfusion was followed for a period of $4 \mathrm{~h}$. Ozone was injected intraperitoneally and epididymally $1.5 \mathrm{~h}$ prior to reperfusion in the intraperitoneal and intra-testicular ozone groups, respectively. Four hours after reperfusion, bilateral orchiectomy was performed for histopathologic assessment for testicular injury and spermatogenesis using Johnsen Score, immune-histochemical staining for endothelial nitric oxide synthase (e-NOS) and inducible nitric oxide synthase (i-NOS) and TUNEL immunohistochemistry for apoptotic cell death. In the T/D group, there was significant testicular damage assessed as atrophic seminiferous tubules, low Johnsen Score, testicular edema, hemorrhage and general pathologic deformations. In the intraperitoneal and intratesticularozone groups, germ cell necrosis was observed in tubules which had incomplete maturation to the level of primary or secondary spermatocytes; however, there was a significant rescue of testicular function and mild to moderate interstitial edema observed. Tissue e-NOS level was significantly lower in both $\mathrm{T} / \mathrm{D}+$ intraperitoneal ozone and T/D + intra-testicular ozone groups compared to the $T / D$ group $(p<0.05$ for each). The $T / D+$ intraperitoneal ozone and $\mathrm{T} / \mathrm{D}+$ intra-testicular ozone groups had similar levels of tissue e-NOS level. Tissue iNOS level was not significantly different between the T/D + intraperitoneal and T/D groups but it was significantly lower in the T/D + intra-testicular ozone group compared to the $T / D$ group $(p \leq 0.01)$. In summary, this study showed that intra-testicular and intraperitoneal ozone application alleviated testicular ischemic damage.

Melatonin administration has been shown to partially restorethe increased oxidative stress as well as the reduced spermatogenic activity level in testes of $I /$ Rrats (Semercioz et al., 2017). A study (Ekici et al., 2012) compared the effect of OTto melatonin in testicular function and morphology in an experimental model of unilateral testicular torsion. Three-month old Wistar rats $(n=24)$ were divided into 4 groups: a sham group where left inguinoscrotal incision was performed, a T/D group (created by rotating the left testis 720 degrees in clockwise direction and fixed within hemiscrotum with an atraumatic silk suture, torsion maintained for $6 \mathrm{~h}$ and then detorsed), melatonin intraperitoneal $+\mathrm{T} / \mathrm{D}$ group and ozone intraperitoneally $(4 \mathrm{mg} / \mathrm{kg})+\mathrm{T} / \mathrm{D}$ group. This was followed by bilateral orchiectomies performed after 1 week and blood and tissue collection for determination of testicular MDA (lead to tubule hemorrhage and germ cell degeneration), inhibin B (as a marker of spermatogenesis), GSH and Nitric Oxide (NO) as well as total plasma Sulfhydryl Group (RSH) (as evaluation of oxidative stress) levels. Significant testicular damage in the T/D group was noted as well as statistically significant differences between the groups for all of studied parameters in the bilateral testes. Testicular MDA increased in T/D group compared to the sham group. Melatonin and ozone treatment suppressed testicular MDA levels. Testicular inhibin B and GSH levels were significantly decreased in the T/D group compared to the sham group, while both melatonin and ozone improved inhibin B and GSH levels to the same extent-ozone known to react with polyunsaturated fatty acids and -SH groups such as GSH. Testicular NO levels were decreased in the melatonin group but significantly increased in the ozone group. Serum RSH significantly decreased in the T/D group compared to the sham group and, although not statistically significant, both melatonin and OT caused further decrease in RSH levels. Finally, melatonin and ozone similarly improved the Johnsen Scores. In sum, that study showed that OT was beneficial in the treatment of testicular torsion and its effects were comparable tomelatonin.

\section{Effect of Ozone on Chemotherapy-Induced Testicular Toxicity}

Testicular toxicity is a frequent adverse effect of cancer chemotherapy with drugs such as Adriamycin (ADR) (Sakai et al., 2018). A study (Salem et al., 2017) assessed the cytoprotective effects of rutin, OT and their 
combination on a known gonadotoxic agent, ADR. In that study, 50 male albino rats were divided into 5 groups: Control group (which received saline + oxygen vehicles), ADR group (which received ADR + saline vehicle+ oxygen vehicles), ADR + rutin group (which received ADR + rutin + oxygen vehicle), ADR + ozone group (which received ADR + ozone + saline vehicle) and finally ADR + rutin + ozone group (which received $\mathrm{ADR}+$ ozone + rutin). The OT was supplemented by rectal insufflations ( 5 applications weekly) at a rate of $0.5 \mathrm{mg} / \mathrm{kg}$ per day for week 1 , then $1 \mathrm{mg} / \mathrm{kg}$ for weeks 2 and 3. ADR was administered by intraperitoneal route 3 times per week for 2 weeks, while rutin was administered at $50 \mathrm{mg} / \mathrm{kg}$ body weight daily intraperitoneally for 3 weeks. All treatments were delivered concomitantly with ADR for 2 weeks and then continued for the third week of the study. Rats were then subjected to overnight fasting and blood withdrawn for hormonal testing that included Luteinizing Hormone (LH), Follicle-Stimulating Hormone (FSH) and testosterone and for other important markers such as MDA as an index of lipid peroxidation, NO, Total Antioxidant Capacity (TAC), C-reactive protein (CRP), Monocytechemotactic Protein-1 (MCP-1) and Leukotriene B4 (LTB4).Both testes and epididymis were excised, weighed and assayed for g-Glutamyltransferase (g-GT), h-Glucuronidase (h-Glu), Alkaline Phosphatase (ALP), Acid Phosphatase (ACP). Sperm function tests including sperm viability, motility, count and morphology were assessed. The results showed that ADR only group had a significant decrease in sperm count, motility, viability and morphology compared to the control group. The ADR+ OT group had 32.8\% increase in sperm count, $29.9 \%$ increase in sperm motility and $45.6 \%$ improvement in sperm morphology compared to ADR only group. The ADR + OT + rutinhad $52.1 \%$ increase in sperm count, $49.1 \%$ increase in sperm motility and $68.4 \%$ improvement in sperm morphologycompared to ADR only group. Additionally, the ADR only grouphad a decrease in serum testosterone levels and significant increase in serum $\mathrm{LH}$ and FSH compared to the control group. The addition of rutin, OT, or combination of rutin + ozone resulted in increased testosterone levels $(48.5 \%, 61.7 \%$ and $100.6 \%$; respectively), reduction of $\mathrm{LH}$ levels $(28.3 \%, 47.8 \%$ and $55.2 \%$; respectively) and reduction of FSH levels (26.2\%, 50.8\% and 54.1\%; respectively) compared to the ADR only group. As for oxidant and antioxidant biomarkers, compared to the control group, ADR increased MDA and NO levels and significantly decreased TAC levels. Compared to ADR only group, rutin, $\mathrm{OT}$, or $\mathrm{OT}+$ rutin resulted in significant decrease of MDA (41\%, $42.9 \%$ and $44.5 \%$; respectively) and NO levels $(50.8 \%, 53.9 \%$ and $57 \%$; respectively) and significant increase of TAC levels $(29.6 \%, 38.5 \%$ and
42.2\%; respectively). As for inflammatory markers, ADR given alone caused a significant increase of CRP, MCP-1 and LTB4 levels compared to the control group. Compared to the ADR only group, rutin, OT and OT + rutin significantly decreased CRP (by $46.9 \%, 42 \%$ and $53.1 \%$; respectively), MCP-1 (54.2\%, 48\% and 60.4\%; respectively) and LTB4 (40.8\%, 39.4\% and 45.1\%; respectively) levels. As for testicular enzymes, the control group showed increase in g-GT, h-Glu, ALP and ACP enzymes compared to the ADR group. The ADR group showed reduction in g-GT (41.9\%) and ALP (36\%) levels compared to the rutin group. The ADR group had significant decrease in g-GT $(46.8 \%)$, h-GLU (44.3\%), ACP (35.4\%) and ALP (38.2\%)levels compared to the OT group. The ADR group had significant decrease in g-GT (49.5\%), h-GLU (49.7\%), ACP (37.1\%) and ALP (44.2\%) levels compared to the rutin + OT group. Taken together, these findings suggest that rutin, OT, or a combination of both, may be protective against ADR toxicity by inhibiting spermatogenic cell damage and enhancing sperm quantity and quality. These phenomena were associated with increased testosterone and antioxidant enzymes levels, decreased testicular enzymes levels of g-GT, hGlu, ACP and ALP and decreased expression of inflammatory markers such as CRP, MCP-1 and LTB4.

\section{Effect of Ozone on Estrogen-Induced Testicular Toxicity}

There are significant concerns about the impact of estrogenic compounds found in the environment on reproductive health, possibly because of environmental accumulation of xenoestrogens (Bouskine et al., 2008; Sikka and Wang, 2008). Exposure to estrogen-like molecules found in the environment, such as wastewater, can negatively affect testicular fetal development, partly by inhibiting spermatogenesis, especially during gestation, when testicular cells are more sensitive to estrogens (Ullah et al., 2018). Ozone can degrade steroids and in particular estradiol (Mills et al., 2015), thus one study (Lassonde et al., 2015) used organ cultures of rat fetal testis as a bioassay to monitor the toxicity of mixture of estrogens: 17B-estradiol (E2), 17 alpha-ethinylestradiol (EE2), estrone (E1) and estriol (E3), at concentrations found in wastewater. The aim of the study was to determine whether OT could remove these compounds and alleviate their potential toxicity. Testes were sampled from fetuses on gestation day 15.5 from time-mated Sprague-Dawley female rats. For each fetus, one testis was placed in control media and the other was treated with a media containing a mixture of 4 estrogens with or without exposure to ozone. Ozonesolutions at doses of $50 \mu \mathrm{g} / \mathrm{L}$ and $500 \mu \mathrm{g} / \mathrm{L}$ were used. The outcomes included immunostaining for AntiMullerian Hormone (AMH), Proliferating Cell Nuclear 
Antigen (PCNA), P450 side chain cleavage, cleavedcaspase 3 , testis and cord volume measurement, Sertoli cells and gonocytes numbers, proliferative index for Sertoli cells and gonocytes and testosterone concentration in culture media. Ozone was able to remove all 4 estrogens below their detection limits with removal efficiencies of $97.5 \%$. The mixture of 4 estrogens induced a significant decrease in the total testis volume likely due a decrease in seminiferous cord volume. However, this negative effect did not occur when ozone was added to the mixture of 4 estrogens. Additionally, the mixture of 4 estrogens significantly decreased the number of gonocytes (stains negative for AMH with large nuclei) per testis compared to controls. The mixture of estrogens decreased the number of Sertolicells (stains positive for AMH and small nuclei). Such harmful outcomes did not occur when ozone was added to the mixture of 4 estrogens. There was no significant effect of the mixture of estrogens before or after ozone treatment on testosterone level in the culture media or on the distribution and morphology of Leydig cells. This study showed that ozone is effective in removing the 4 target estrogens at concentrations below their limit of detection and potentially able to remove the toxicity of this mixture.

\section{Effect of Ozone on Bacteria Present in the Semen}

Ozonides (secondary reaction products of ozone) inactivateseveral microorganisms. A study performed (Gradil et al., 1995) evaluated ozone treatment of bull semen diluted with milk intentionally contaminated with Pseudomonas aeruginosa, Escherichia coli and Campylobacter fetus subsp. venerealis. That study also determined the optimal concentration and contact time with the maximal bactericidal results and minimal effect on sperm motility. Bacterial isolates with Pseudomonas aeruginosa, Escherichia coli and Campylobacter fetus subsp. venerealis were obtained and ozonation of semen diluent was performed. Thus in fresh bull semen, Pseudomonas aeruginosa, Escherichia coli and Campylobacter fetus subsp. venerealis were added to the ozonized ( $3 \mathrm{~min}$ at $5,10,15$, or $20 \mu \mathrm{g} / \mathrm{ml}$ or $5 \mathrm{~min}$ at 20 $\mu \mathrm{g} / \mathrm{ml})$ or oxygenized (3-5 $\mathrm{min}$ at $\left.0 \mathrm{ug} \mathrm{O}_{3} / \mathrm{mL}\right)$ milk diluent (consisting of 1 part semen to 10 parts milk). Milk diluent containing semen without bacteria was used as a control. Sperm motility was assessed after $10 \mathrm{~min}$. Two and $18 \mathrm{~h}$ after cooling, semen was evaluated for motility and cultured for microorganisms. The results showed that bacteria were not detected in the controls and that Pseudomonas aeruginosa, Escherichia coli and Campylobacter fetus subsp. venerealis were inactivated by ozone concentration at 2 and $18 \mathrm{~h}$. The bactericidal activity of ozone increased with increasing ozone concentration. Ozone exposure for $2 \mathrm{~h}$ at $5 \mu \mathrm{g} / \mathrm{mL}$ concentration revealed that Pseudomonas aeruginosa and Escherichia coli were more resistant than Campylobacter fetus subsp. venerealis. These results indicated that ozone application could inactivate microbial agents in bovine semen and that direct ozonation of diluted semen was spermicidal as low as 5 $\mu \mathrm{g} / \mathrm{mL}$ concentrations of ozone.

\section{Oxidative Stress, ROS, Ozone and Male Reproduction}

Oxidative Stress (OS) occurs when the balance between ROS and antioxidant scavengers is disrupted. OS is associated with reproductive decline in males and represents a significant biological mechanism accompanying ageing as well as chronic conditions associated with ageing. On the other hand, OS plays a key role in regulating several molecular pathways that are important for maintaining homeostasis at the level of the cells, tissues, organs and systems. Therefore, a fine balance between elements favoring OS and those that remove ROS by scavenging is necessary for the physiology of the male reproductive function. In the male reproductive system, ROS are crucialmolecular species responsible for physiological functions that include spermatogenesis, epididymal transport, maturation of spermatozoa and post-ejaculation phenomena such as motility, capacitation and the acrosome reaction. It appears that there is a correlation between the OS that is associated with ageing and a number of conditions ultimately leading to male reproductive system dysfunctions that include reduction of the potential for fertility because of decline of the quality of semen as well as alterations of endocrine functions. The role of ROS and OS in regulating male fertility, with particular focus on the association between ageing and fertility, assumes particular relevance considering the trend toward increased life expectancy and paternal age. Combination of these two factors implies increased risk for fertility, complications of pregnancy and decreased potential for health in descendants. Therefore, it is of utmost importance to assess the role of OS, ROS and antioxidants as key players in influencing male reproductive physiology and its sequels. In this context, the role of antioxidants, such as for example, supplements based on vitamins $\mathrm{C}$ and $\mathrm{E}$, assumes interesting contours; on one side, they are proposed as tools to support male fertility and reproductive health. On the other side, superfluous use of antioxidants may lead to the opposite outcomes by reducing physiologic formation of ROS, species that play essential roles in male reproductive physiology (Leisegang et al., 2017).

If unnecessary supplementation with antioxidants is not to be recommended in the context of male reproductive physiology, it is also true that a balanced diet containing antioxidants positively contributes to 
semen quality and fertility. A recent systematic review of 1,944 scientific articles on this topic concluded that "healthy diets rich in some nutrients such as omega-3 fatty acids, some antioxidants (vitamin E, vitamin $C, \beta$ carotene, selenium, zinc, cryptoxanthin and lycopene), other vitamins (vitamin D and folate) and low in saturated fatty acids and trans-fatty acids were inversely associated with low semen quality parameters" (Salas-Huetos et al., 2017). To this end, one of us (MR), has developed an ad hoc formulated probiotic containing antioxidants and other essential nutrients that aims at regulating Klotho, a gene coding for a protein known to delay ageing and contributing to healthy reproductive physiology (Ruggiero, 2018). Preliminary observation seems to indicate net positive results and it can be hypothesized that OT together with this highly specific nutritional approach may yield significant result in supporting male reproductive health. Thus, the effectiveness of Klotho in attenuating DNA damage inflicted by oxidants may prove useful also in those conditions where toxicants induce OS that, in turn, is responsible for damage to nucleic acids and authophagy of germ cells (Chen et al., 2018). Given the importance of fine balance between the effects of antioxidants and physiologic ROS production in determining the health of the male reproductive function, it can be speculated that OT may serve not only as bona fide "therapy" but also as a preventive approach to support healthy male reproductive function in conditions where high doses of antioxidants have to be used. Such an approach may require preliminary determination of ROS synthesis and OS by chemiluminescence, even though novel, specific methods for ROS determination in the male reproductive tract will have to be developed since most analytical methods for ROS refer to peripheral tissues (Liu et al., 1996).

Importance of the delicate balance between ROS and OS in the male reproductive tract is evident in the case of varicocele; according to a study performed by authors from the Cleveland Clinic Foundation of Ohio, USA "Patients with varicocele had significantly higher reactive oxygen species levels than controls $(p=0.02)$. Reactive oxygen species levels did not differ significantly between infertile and men with incidental varicocele. Total antioxidant levels were significantly lower among men with varicocele $(p=0.02)$ and those with incidental varicocele compared to controls $(\mathrm{p}=$ 0.05)." Consistent with these results, the Authors concluded that "These changes may be related to functional sperm abnormalities and infertility seen commonly in these patients" (Hendin et al., 1999). OT may prove useful in preventing testicular damage in patients with varicocele treated with antioxidants in a manner similar to what recently demonstrated in an experimental cryptorchid animal model (Biçer et al., 2018). Likewise, because of its known antimicrobial properties that include antiviral activities (Brié et al., 20118), OT may prove useful in preventing testicular damage inflicted by viral infections such as mumps. Rationale for the use of OT in male reproductive health requires precise determination of OS in clinical practice and several types of laboratory analyses have been devised to estimate OS in semen. These assays can be divided in two major categories; dicrect assays that evaluate the degree of oxidation inside a sperm cell and indirect assays that provide for an estimate of the damaging effects of OS. Chemiluminescence, flow cytometry, use of nitroblue tetrazolium and cytochrome c reduction constitute examples of direct assays; the myeloperoxidase test and measurements of lipid peroxidation, oxidation-reduction potential and total antioxidant capacity are among the indirect assays (Agarwal and Majzoub, 2017).

\section{Conclusion}

The scientific literature available thus far shows that most studies pertaining to the effect of OT on male reproduction were performed in animals (Table 1). There is a definite need for the development of at least somepilot studies in humans using OT in its safest route of administration, which is probably the transdermal route. This route of administration exploits the features of the newly described organ termed "interstitium" (Benias et al., 2018), that is an organ that connects the skin with deeper structures such as the lymphatic and nervous systems. In addition, such a way of administration may be complemented by ad hoc developed formulas targeting the lymphatic andautonomic systems, the latter being connected to the testis through the blood vessels of the spermatic cord, the mesorchium and the ligament to us bridge between epididymal tail and testis (Wrobel and Abu-Ghali, 1997). It was recently observed that lymphatic drainage and autonomic stimulation using a conceptually similar approach proved effective in treating symptoms of autism (Antonucci et al., 2019) and synergy with OT in the context of male reproduction conditions may be hypothesized. In this optic, design of future studies would include men with poor semen analyses in order to assess whether transdermal ozone would improve semen parameters. This is of particular relevance considering that male factor infertility constitutes up to $30 \%$ of couple infertility and it is very common that semen parameters are not reversed bymedical or surgical treatments such as varicocele repair or vasectomy reversal. 
Table 1: Summary of the pertinent studies presented in the manuscript

Pre-term

\begin{tabular}{|c|c|}
\hline Methods & Measured variables \\
\hline $\begin{array}{l}\text { Study by Tusat et al. }(2017) \\
\text {-Rat model } \\
\text {-Torsion/detorsion (T/D) } \\
\text { +/- medical ozone therapy (OT) } \\
\text { intraperitoneal }\end{array}$ & $\begin{array}{l}\text {-Biochemical parameters: Ischemia Modified } \\
\text { Albumin (IMA), Total Antioxidant Status } \\
\text { (TAS), Total Oxidant Status (TOS) and } \\
\text { Oxidative Stress Index (OSI). } \\
\text {-Testicular Biopsy for Johnsen Score to } \\
\text { assess testicular tissue injury. } \\
\text {-IMA levels determined by calorimetric } \\
\text { method } \\
\text {-TOS and TAS levels using calorimetric kits. }\end{array}$ \\
\hline $\begin{array}{l}\text { Study by Salem et al. (2017) } \\
\text {-Rat model } \\
- \text { Adriamycin }(A D R) \pm \text { ozone } \\
-A D R \pm \text { rutin } \\
-A D R \pm \text { ozone and rutin }\end{array}$ & $\begin{array}{l}\text {-Sperm function tests: sperm viability, } \\
\text { motility, count and morphology. } \\
\text {-Biochemical tests: LH, FSH, testosterone, } \\
\text { g-GT, h-Glu, ALP, ACP, MDA, NO, TAC, } \\
\text { CRP, MCP-1 and LTB4. }\end{array}$ \\
\hline
\end{tabular}

Study by Mete et al. (2017)

-Rat model

-Torsion/Detorsion (T/D) \pm

Intratesticular (IT) ozone therapy.

Study by Lassonde et al. (2015)

-Rat model

-4 estrogen hormones + varying

ozone concentrations

-Control water: equal volume

of alcohol and ozone.

-One testes in control and the

other in ozone

Study by Ekici et al. (2012)

-Rat model

-Torsion/detorsion (TD) \pm

ozone \pm melatonin
Intraperitoneal (IP) ozone \pm

-Testicular Biopsy for Johnsen Score to assess testicular injury.

-TUNEL indirect immunohistochemical staining to measure apoptotic cell death.

-Immunohistochemistry for $\mathrm{AMH}$,

PCNA, p450 and CC-3.

-Testis and cord volume measured

-Apoptotic cells measured by number

of CC-3 positive cells.

-Testosterone assay using ELISA kit.

-Markers measured: MDA, Inhibin B,

GSH, RSH and NO.

-Histopathologic evaluation: Johnsen Score for testicular injury and spermatogenesis.

Study by Gradil et al. (1995) -In fresh bovine semen, P. aeruginosa, Campylobacter and $E$. Coli were added to the ozonized or oxygenized milk diluent.

-Control: milk diluent + semen without the bacteria.

-Study assessed ozone treatment of milk semen diluents and effects in controlling $P$. aeruginosa, E. coli and Campylobacter in bull semen.

-Determined concentration and contact time with maximum bactericidal results and minimal effect on sperm motility.
Results Conclusions
-IMA, TOS, and OSI significantly higher in T/D vs. control while OT significantly reduced all levels. No significant difference in TAS level. $-\mathrm{T} / \mathrm{D}+\mathrm{OT}$ had seminiferous tubule more regular vs. T/D group. Germinal epithelial cells: regular alignment in lumen and spermatozoa partly observed

\section{-Sperm Dynamics}

- ADR decreased sperm count, motility, viability and morphology.

-Adding ozone reversed the effect of ADR

-Biochemical test

- ADR only: TAC and testosterone decreased.

LH, FSH, g-GT, h-Glu, ALP, ACP, NO, MDA, CRP, MCP-1 and LTB4 increased.

$-\mathrm{ADR} \pm$ rutin + ozone reversed the effect.

-T/D group: significant testicular damage and lower JS scores.

-IT and IP ozone groups:

a) Tubules with germ cell necrosis and

incomplete maturation.

b) Rescue of testicular function.

-Ozone removed all 4 estrogens below their detection limit.

-No effect on testosterone secretion

-Decrease in Sertoli cells and gonocytes

due to greater apoptosis

-Testicular damage in the T/D group. -T/D increased NO and MDA. RSH decreased after T/D.

- Ozone and melatonin increased GSH and inhibin B, decreased MDA, NO and RSH -Ozone and melatonin improved Johnsen Score.

-Medical OT reduced oxidative stress markers and was protective in testicular torsion.

Rutin, ozone, or combination of both protect from ADR toxicity. -Rutin, ozone, or combination of both may protect against spermatogenic cell damage and enhance quantity/quality of spermatogenesis.

-Ozone therapy, both IP and IT, can alleviate testicular ischemic damage due to T/D

-Negative effect of estrogens on testicular development; an effect that was not observed with ozone application.

-Ozone therapy was beneficial in treatment of testicular torsion and its effects were comparable to melatonin.

-P. aeruginosa, E. coli and Campylobacter inactivated by ozone

-Bactericidal activity increased with increasing ozone.

-P. aeruginosa and E. coli more resistant than Campylobacter to ozone.

Abbreviations: ACP: Acid Phosphatase, ADR: Adriamycin, AI: Apoptotic Index, ALP: alkaline phosphatase , AMH: Anti-Mullerian Hormone, B/l: Bilateral, CC-3: Cleaved-Caspase 3-positive cells, C: Celsius, CO: Carbon Monoxide, CRP: C-Reactive Protein, Dec: Decrease, g-GT: g-Glutamyltransferase, h-Glu: h-glucuronidase, IHC: immunohistochemistry, IMA: Ischemia Modified Albumin, i-NOS: inducible Nitric Oxide Synthase, IP: Intraperitoneal, IR: Immunoreactivity, IVCYC: Intravenous Cyclophosphamide, L: Liter, LTB4: Leukotriene B4, MCP-1: brain Monocyte Chemotactic Protein-1, MDA: Malondialdehyde, NO: Nitrates, NO: Nitric Oxide, $\mathrm{O}_{2}$ : Oxygen, $\mathrm{O}_{3}$ : Ozone, OSI: oxidative stress index, OT: ozone therapy, P450: cytochrome p450, PCNA: Proliferating Cell Nuclear Antigen, PM10: Particular Matter, SO ${ }_{2}$ : Sulfur dioxide, TAC: Total Antioxidant Capacity, TAS: Total Antioxidant Status, TOS: Total Oxidant Status, TT: Testicular Torsion

\section{Acknowledgement}

There were no funds used or paid to any of the authors for writing this manuscript.

\section{Author's Contribution}

The authors contributed to the genesis, development and writing of the topic described in this manuscript.
Zaher Merhi, Ali Bazzi and John Zhang: Performed literature search, wrote the first drafts of the manuscript, made the figure and the table and approved the final version of the manuscript.

Rajean Moseley-LaRue, Amber Ray Moseley and André Hugo Smith: Contributed to the development of the topic and contributed to the writing of the manuscript and edits to the figure and table.

Marco Ruggiero: Contributed to the final version of this manuscript. 


\section{Ethics}

This article is an original review article based on previously published papers and does not contain new original data.

\section{Conflict of Interest}

A.H.S. is the president of HOCATT USA.

A.R.M is the Chief Executor Officer of HOCATT USA.

R.M.L is the Chief Operational Officer and Medical Director of HOCATT USA.

Z.M. acts as a paid consultant for HOCATT USA and has received a free HOCATT machine from A.R.M and A.H.S. in order to perform a recently IRB-approved study pertaining to the effect of ozone therapy on female and male fertility. He was not paid to write this manuscript.

J.Z. and A.B. have nothing to disclose.

M.R. is the founder and CEO of the Swiss company Silver Spring Sagl, a company that produces and distributes foods and supplements; none of the products of this company is mentioned in this article. M.R. is member of the Editorial Board of The American Journal of Immunology and is waived from the Article Processing fee for this contribution; he receives no remuneration for his editorial work.

\section{Advisory}

No information in this paper is presented by the authors as medical advice. Caregivers, researchers and interested parties should research all information given. Beginning any significant biomedical or other interventions that may impact physiology or making changes to an established regimen should be discussed with the patient's physician in advance. Standard of care for each pathology must be followed as well as rules and regulations established by Health Authorities of each Country.

\section{References}

Agarwal, A. and A. Majzoub, 2017. Laboratory tests for oxidative stress. Ind. J. Urol., 33: 199-206. DOI: 10.4103/iju.IJU_9_17

Antonucci, N., S. Pacini and M. Ruggiero, 2019. Clinical experience of integrative autism treatment with manual lymphatic drainage. EC Neurol., 11: 15-22.

Benias, P.C., R.G. Wells, B. Sackey-Aboagye, H. Klavan and J. Reidy et al., 2018. Structure and distribution of an unrecognized interstitium in human tissues. Sci. Rep., 8: 4947-4947.

DOI: $10.1038 / \mathrm{s} 41598-018-23062-6$

Beyaz, S.G. and H. Sayhan, 2018. Six-month results of cervical intradiscal oxygen-ozone mixture therapy on patients with neck pain: Preliminary findings. Pain Phys., 21: E449-E456.
Biazzo, A., A.S. Corriero and N. Confalonieri, 2018. Intramuscular oxygen-ozone therapy in the treatment of low back pain. Acta Bio-Med.: Atenei Parmensis, 89: 41-46.

Biçer, Ş., C. Gürsul, İ. Sayar, O. Akman and S. Çakarlı et al., 2018. Role of ozone therapy in preventing testicular damage in an experimental cryptorchid rat model. Med. Sci. Monitor Basic Res., 24: 5832-5839. DOI: 10.12659/MSM.910459

Bocci, V., G. Valacchi, F. Corradeschi and G. Fanetti, 1998. Studies on the biological effects of ozone: 8 . Effects on the total antioxidant status and on interleukin-8 production. Mediators inflammat., 7: 313-317.

Bocci, V.A., 2006. Scientific and medical aspects of ozone therapy. State of the art. Arch. Med. Res., 37: 425-435. DOI: 10.1016/j.arcmed.2005.08.006

Bocci, V.A., 2007. Can ozonetherapy be performed if the biochemistry of the process cannot be controlled? Arch. Med. Res., 38: 584-585. DOI: 10.1016/j.arcmed.2007.03.005

Bocci, V.A., 2008. Why orthodox medicine has not yet taken advantage of ozone therapy. Arch. Med. Res., 39: 259-260. DOI: $10.1016 /$ j.arcmed.2007.09.007

Bouskine, A., M. Nebout, B. Mograbi, F. Brucker-Davis and C. Roger et al., 2008. Estrogens promote human testicular germ cell cancer through a membranemediated activation of extracellular regulated kinase and protein kinase A. Endocrinology, 149: 565-573. DOI: $10.1210 /$ en.2007-1318

Brié, A., N. Boudaud, A. Mssihid, J. Loutreul and I. Bertrand et al., 2018. Inactivation of murine norovirus and hepatitis $A$ virus on fresh raspberries by gaseous ozone treatment. Food Microbiol., 70: 1-6. DOI: 10.1016/j.fm.2017.08.010

Buyuklu, M., F.M. Kandemir, T. Set, E.M. Bakirci and H. Degirmenci et al., 2017. Beneficial effects of ozone therapy on oxidative stress, cardiac functions and clinical findings in patients with heart failure reduced ejection fraction. Cardiovascular Toxicol., 17: 426-433. DOI: 10.1007/s12012-017-9400-8

Carpendale, M.T., J. Freeberg and J.M. Griffiss, 1993. Does ozone alleviate AIDS diarrhea? J. Clin. Gastroenterol., 17: 142-145.

DOI: 10.1097/00004836-199309000-00010

Chen, N., M. Lin, N. Liu, S. Wang and X. Xiao, 2018. Methylmercury-induced testis damage is associated with activation of oxidative stress and germ cell autophagy. J. Inorganic Biochem., 190: 67-74. DOI: 10.1016/j.jinorgbio.2018.10.007

Cicerone, R.J., 1987. Changes in stratospheric ozone. Science, 237: 35-42.

DOI: $10.1126 /$ science. 237.4810 .35 
Clavo, B., N. Santana-Rodriguez, P. Llontop, D. Gutierrez and G. Suarez et al., 2018. Ozone therapy as adjuvant for cancer treatment: Is further research warranted? Evidence-Based Complementary Alternative Med.

Cooke, E.D., A.G. Pockley, A.T. Tucker, J.D. Kirby and A.E. Bolton, 1997. Treatment of severe Raynaud's syndrome by injection of autologous blood pretreated by heating, ozonation and exposure to ultraviolet light (H-O-U) therapy. Int. Angiol.: J. Int. Un. Angiol., 16: 250-254.

Costa, T., D. Linhares, M. Ribeiro da Silva and N. Neves, 2018. Ozone therapy for low back pain. A systematic review. Acta Reumatol. Portuguesa, 43: 172-181.

Di Paolo, N., E. Gaggiotti and F. Galli, 2005. Extracorporeal blood oxygenation and ozonation: Clinical and biological implications of ozone therapy. Redox Report: Commun. Free Rad. Res., 10: 121-130. DOI: 10.1179/135100005X38888

Dogan, R., A.M. Hafiz, H.S. Kiziltan, A. Yenigun and N. Buyukpinarbaslili et al., 2018. Effectiveness of radiotherapy + ozone on tumoral tissue and survival in tongue cancer rat model. Auris Nasus Larynx, 45: 128-134. DOI: 10.1016/j.anl.2017.03.017

Dokmeci, D., 2006. Testicular torsion, oxidative stress and the role of antioxidant therapy. Folia Med., 48: 16-21.

Duricic, D., H. Valpotic and M. Samardzija, 2015. Prophylaxis and therapeutic potential of ozone in buiatrics: Current knowledge. Anim. Reproduct. Sci., 159: 1-7. DOI: 10.1016/j.anireprosci.2015.05.017

Ekici, S., A.I. Dogan Ekici, G. Ozturk, F. Benli Aksungar and O. Sinanoglu et al., 2012. Comparison of melatonin and ozone in the prevention of reperfusion injury following unilateral testicular torsion in rats. Urology, 80: 899-906. DOI: 10.1016/j.urology.2012.06.049

Evstigneeva, I.S., A.G. Kulikov, O.V. Yarustovskaya, A.S. Shakhova and M.G. Lutoshkina, 2018. [Physical therapy of psoriasis. The modern methodological approaches]. Voprosy Kurortologii Fizioterapii Lechebnoi Fizicheskoi Kultury, 95: 53-58. DOI: 10.17116/kurort201895153-58

Fitzpatrick, E., O.J. Holland and J.J. Vanderlelie, 2018. Ozone therapy for the treatment of chronic wounds: A systematic review. Int. Wound J., 15: 633-644. DOI: 10.1111 iwj.12907

Frankum, B. and C.H. Katelaris, 1993. Ozone therapy in AIDS--truly innocuous? Med. J. Australia, 159: 493-493.

Garber, G.E., D.W. Cameron, N. Hawley-Foss, D. Greenway and M.E. Shannon, 1991. The use of ozone-treated blood in the therapy of HIV infection and immune disease: A pilot study of safety and efficacy. AIDS, 5: 981-984.

DOI: $10.1097 / 00002030-199108000-00009$
Gloor, M. and B.A. Lipphardt, 1976. [Studies on ozone therapy of acne vulgaris]. Zeitschrift fur Hautkrankheiten, 51: 97-101.

Gradil, C., M.D. Eaglesome, B. Stewart, M.M. Garcia and F. Quimby, 1995. Bactericidal effects of ozone at nonspermicidal concentrations. Canad. J. Vet. Res., 59: 183-186.

Hendin, B.N., P.N. Kolettis, R.K. Sharma, A.J. Thomas $\mathrm{Jr}$ and A. Agarwal, 1999. Varicocele is associated with elevated spermatozoal reactive oxygen species production and diminished seminal plasma antioxidant capacity. J. Urolol., 161: 1831-1834. DOI: $10.1016 / \mathrm{S} 0022-5347(05) 68818-0$

Hidalgo-Tallon, J., S. Menendez-Cepero, J.S. Vilchez, C.M. Rodriguez-Lopez and E.P. Calandre, 2013. Ozone therapy as add-on treatment in fibromyalgia management by rectal insufflation: An open-label pilot study. J. Alternative Complementary Med., 19: 238-242. DOI: 10.1089/acm.2011.0739

Hu, B., J. Zheng, Q. Liu, Y. Yang and Y. Zhang, 2018. The effect and safety of ozone autohemotherapy combined with pharmacological therapy in postherpetic neuralgia. J. Pain Res., 11: 1637-1643. DOI: $10.2147 /$ JPR.S154154

Inal, M., A. Dokumacioglu, E. Ozcelik and O. Ucar, 2011. The effects of ozone therapy and coenzyme $\mathrm{Q}(1)(0)$ combination on oxidative stress markers in healthy subjects. Irish J. Med. Sci., 180: 703-707. DOI: $10.1007 / \mathrm{s} 11845-011-0675-7$

Karatieieva, S., I. Plesh, O. Yurkiv, S. Semenenko and I. Kozlovskaya, 2017. New method of treatment of pyoinflammatory soft tissue complications in patients with diabeted mellitus. Georgian Med. News.

Kashiwagi, K., K. Saito, Y.D. Wang, H. Takahashi and K. Ishijima et al., 2001. Safety of ozonated solution as an antiseptic of the ocular surface prior to ophthalmic surgery. Int. J. Ophthalmol., 215: 351-356. DOI: $10.1159 / 000050884$

Kaya, A., M. Sonmez, T. Kar, A. Haholu and Y. Yildirim et al., 2017. Efficiency of ozone therapy in a rat model of experimental uveitis. Ocular Immunol. Inflammat., 25: 695-700. DOI: $10.3109 / 09273948.2016 .1161057$

Kosheleva, I.V. and A.G. Kulikov, 2001. [Effectiveness of complex therapy of eczema patients with the use of various methods of ozone therapy]. Voprosy Kurortol.ogii Fizioterapii Lechebnoi Fizicheskoi Kultury.

Kucukgul, A., S. Erdogan, R. Gonenci and G. Ozan, 2016. Beneficial effects of nontoxic ozone on $\mathrm{H}_{2} \mathrm{O}_{2}$ induced stress and inflammation. Biochem. Cell Biol., 94: 577-583. DOI: 10.1139/bcb-2016-0033

Kushmakov, R., J. Gandhi, O. Seyam, W. Jiang and G. Joshi et al., 2018. Ozone therapy for diabetic foot. Med. Gas Res., 8: 111-115. DOI: $10.4103 / 2045-9912.241076$ 
Lassonde, G., D. Nasuhoglu, J.F. Pan, B. Gaye and V. Yargeau et al., 2015. Ozone treatment prevents the toxicity of an environmental mixture of estrogens on rat fetal testicular development. Reprod. Toxicol., 58: 85-92. DOI: $10.1016 /$ j.reprotox.2015.09.001

Leisegang, K., R. Henkel and A. Agarwal, 2017. Redox regulation of fertility in aging male and the role of antioxidants: A savior or stressor. Curr. Pharmaceutical Design, 23: 4438-4450. DOI: $10.2174 / 1381612822666161019150241$

Li, B., C. Liu, L. Wang, Y. Li and Y. Du et al., 2018. The value of ozone in CT-guided drainage of multiloculated pyogenic liver abscesses: A randomized controlled study. Canad. J. Gastroenterol. Hepatol., 2018: 1236974-1236974. DOI: $10.1155 / 2018 / 1236974$

Liu, L., C. Dahlgren, H. Elwing and H. Lundqvist, 1996. A simple chemiluminescence assay for the determination of reactive oxygen species produced by human neutrophils. J. Immunol. Meth., 192: 173-178.

DOI: 10.1016/0022-1759(96)00049-X

Madej, P., A. Plewka, J.A. Madej, D. Plewka and W. Mroczka et al., 2007. Ozone therapy in induced endotoxemic shock. II. The effect of ozone therapy upon selected histochemical reactions in organs of rats in endotoxemic shock. Inflammation, 30: 69-86.

Manoto, S.L., M.J. Maepa and S.K. Motaung, 2018. Medical ozone therapy as a potential treatment modality for regeneration of damaged articular cartilage in osteoarthritis. Saudi J. Biol. Sci., 25: 672-679. DOI: 10.1016/j.sjbs.2016.02.002

Mete, F., H. Tarhan, O., Celik, I. Akarken and K. Vural et al., 2017. Comparison of intraperitoneal and intratesticular ozone therapy for the treatment of testicular ischemia-reperfusion injury in rats. Asian J. Androl., 19: 43-46.

Mills, M.R., K. Arias-Salazar, A. Baynes, L.Q. Shen and J. Churchley et al., 2015. Removal of ecotoxicity of 17alpha-ethinylestradiol using TAML/peroxide water treatment. Scientific Rep., 5: 10511-10511. DOI: $10.1038 /$ srep 10511

Muminov, A.I. and N. Khushvakova, 2002. [Ozone therapy in patients with chronic purulent frontal sinusitis]. Vestnik Otorinolaringologii.

OPOI, 1994. Oxygen plus ozone inactivate HIV. AIDS Patient Care, 8: 167-167. PMID: 11362147

Ozturk, O., H.A. Eroglu, S. Ustebay, M. Kuzucu and Y. Adali, 2018. An experimental study on the preventive effects of $\mathrm{N}$-acetyl cysteine and ozone treatment against contrast-induced nephropathy. Acta Cirurgica Brasileira, 33: 508-517. DOI: $10.1590 / \mathrm{s} 0102-865020180060000005$
Raeissadat, S.A., S.M. Rayegani, B. Forogh, P. Hassan Abadi and M. Moridnia et al., 2018. Intraarticular ozone or hyaluronic acid injection: Which one is superior in patients with knee osteoarthritis? A 6-month randomized clinical trial. J. Pain Res., 11: 111-117.

DOI: $10.2147 /$ JPR.S142755

Resitoglu, B., Y. Celik, M. Komur, A. Polat and S. Erdogan et al., 2018. The efficacy of ozone therapy in neonatal rats with hypoxic ischemic brain injury. Bratislavske Lekarske Listy, 119: 81-85. DOI: 10.4149/BLL 2018015

Ruggiero, M., 2018. A novel approach to Klotho aimed at delaying and reversing aging. Bio Accent Open J. HIV, 3: 029-029.

Safwat, M.H., M.M. El-Sawalhi, M.N. Mausouf and A.A. Shaheen, 2014. Ozone ameliorates age-related oxidative stress changes in rat liver and kidney: Effects of pre- and post-ageing administration. Biochemistry, 79: 450-458. DOI: $10.1134 / \mathrm{S} 0006297914050095$

Sakai, K., M. Ideta-Otsuka, H. Saito, Y. Hiradate and K. Hara et al., 2018. Effects of doxorubicin on sperm DNA methylation in mouse models of testicular toxicity. Biochem. Biophys. Res. Commun., 498: 674-679.

DOI: $10.1016 /$ j.bbrc.2018.03.044

Salas-Huetos, A., M. Bulló and J. Salas-Salvadó, 2017. Dietary patterns, foods and nutrients in male fertility parameters and fecundability: A systematic review of observational studies. Human Reprod. Update, 23: 371-389. DOI: $10.1093 /$ humupd/dmx006

Salem, E.A., N.A. Salem and W.J. Hellstrom, 2017. Therapeutic effect of ozone and rutin on adriamycininduced testicular toxicity in an experimental rat model. Andrologia, 49: e12603-e12603.

DOI: $10.1111 /$ and.12603

Sato, H., Y. Wananabe and H. Miyata, 1990. Virucidal effect of ozone treatment of laboratory animal viruses. Jikken dobutsu. Exp. Anim., 39: 223-229. DOI: 10.1538/expanim1978.39.2_223

Semercioz, A., A.K. Baltaci, R. Mogulkoc and M.C. Avunduk, 2017. Effect of zinc and melatonin on oxidative stress and serum inhibin-b levels in a rat testicular torsion-detorsion model. Biochem. Genet., 55: 395-409. DOI: 10.1007/s10528-017-9826-5

Seyam, O., N.L. Smith, I. Reid, J. Gandhi and W. Jiang et al., 2018. Clinical utility of ozone therapy for musculoskeletal disorders. Med. Gas Res., 8: 103-110. DOI: $10.4103 / 2045-9912.241075$

Shamim, T., 2017. Ozone therapy in dentistry: revisited. Med. Gas Res., 7: 278-278.

DOI: $10.4103 / 2045-9912.222453$ 
Shimizu, S., P. Tsounapi, F. Dimitriadis, Y. Higashi and T. Shimizu et al., 2016. Testicular torsiondetorsion and potential therapeutic treatments: A possible role for ischemic postconditioning. Int. J. Urol., 23: 454-463. DOI: 10.1111/iju.13110

Sikka, S.C. and R. Wang, 2008. Endocrine disruptors and estrogenic effects on male reproductive axis. Asian J. Androl., 10: 134-145. DOI: $10.1111 / \mathrm{j} .1745-7262.2008 .00370 . \mathrm{x}$

Smith, N.L., A.L. Wilson, J. Gandhi, S. Vatsia and S.A. Khan, 2017. Ozone therapy: An overview of pharmacodynamics, current research and clinical utility. Med. Gas Res., 7: 212-219. DOI: $10.4103 / 2045-9912.215752$

Steinhart, H., S. Schulz and R. Mutters, 1999. Evaluation of ozonated oxygen in an experimental animal model of osteomyelitis as a further treatment option for skull-base osteomyelitis. Eur. Arch. Oto-RhinoLaryngol., 2561: 153-157. DOI: $10.1007 / \mathrm{s} 004050050130$

Sugita, H., T. Asai, K. Hayashi, T. Mitsuya and K. Amanuma et al., 1992. Application of ozone disinfection to remove Enterococcus seriolicida, Pasteurella piscicida and Vibrio anguillarum from seawater. Applied Environ. Microbiol., 58: 4072-4075. PMID: 1476447

Tasdemir, C., S. Tasdemir, N. Vardi, B. Ates and Y. Onal et al., 2013. Evaluation of the effects of ozone therapy on Escherichia coli-induced cytitis in rat. Irish J. Med. Sci., 182: 557-563. DOI: $10.1007 / \mathrm{s} 11845-013-0926-\mathrm{x}$

Tezcan, A.H., O. Ozturk, S. Ustebay, Y. Adali and H. Yagmurdur, 2018. The beneficial effects of ozone therapy in acetaminophen-induced hepatotoxicity in mice. Pharmacol. Reports, 70: 340-345. DOI: $10.1016 /$ j.pharep.2017.11.003

Turner, C.R., S.R. Kleeberger and E.W. Spannhake, 1989. Preexposure to ozone blocks the antigeninduced late asthmatic response of the canine peripheral airways. J. Toxicol. Environ. Health, 28: 363-371. DOI: 10.1080/15287398909531355
Tusat, M., A. Mentese, S. Demir, A. Alver and M. Imamoglu, 2017. Medical ozone therapy reduces oxidative stress and testicular damage in an experimental model of testicular torsion in rats. Int. Braz. J., 43: 1160-1166. DOI: $10.1590 / \mathrm{s} 1677-5538 . i b j u .2016 .0546$

Ullah, A., M. Pirzada, S. Jahan, H., Ullah and G. Shaheen et al., 2018. Bisphenol A and its analogs bisphenol B, bisphenol $\mathrm{F}$ and bisphenol $\mathrm{S}$ : Comparative in vitro and in vivo studies on the sperms and testicular tissues of rats. Chemosphere, 209: 508-516.

DOI: $10.1016 /$ j.chemosphere.2018.06.089

Wang, Z., Q. Han, Y.L. Guo,, X.H. Liu and T. Qiu, 2018. Effect of ozone oxidative preconditioning on inflammation and oxidative stress injury in rat model of renal transplantation. Acta Cirurgica Brasileira, 33: 238-249. DOI: $10.1590 / \mathrm{s} 0102-865020180030000006$

Wrobel, K.H. and N. Abu-Ghali, 1997. Autonomic innervation of the bovine testis. Acta Anatomica, 160: 1-14. DOI: 10.1159/000147991

Wu, M.Y., C.Y. Xing, J.N. Wang, Y. Li and X.W. Lin et al., 2018. Therapeutic dosage of ozone inhibits autophagy and apoptosis of nerve roots in a chemically induced radiculoneuritis rat model. Eur. Rev. Med. Pharmacol. Sci., 22: 1787-1797.

Yamayoshi, T. and N. Tatsumi, 1993. Microbicidal effects of ozone solution on methicillin-resistant Staphylococcus aureus. Drugs Under Exp. Clin. Res., 19: 59-64. PMID: 8223143

Yu, M., Y. Zhao and X. Zhang, 2018. Gardenoside combined with ozone inhibits the expression of $\mathrm{P} 2 \mathrm{X} 3$ and $\mathrm{P} 2 \mathrm{X} 7$ purine receptors in rats with sciatic nerve injury. Molecular Med. Rep., 17: 7980-7986. DOI: $10.3892 / \mathrm{mmr} .2018 .8803$

Zanardi, I., E. Borrelli, G. Valacchi, V. Travagli and V. Bocci, 2016. Ozone: A multifaceted molecule with unexpected therapeutic activity. Curr. Med. Chem., 23: 304-314.

DOI: $10.2174 / 0929867323666151221150420$ 